\title{
Inferring neuronal functional connectivity using dynamic Bayesian
} networks

\author{
Seif Eldawlatly*1, Yang Zhou ${ }^{2}$, Rong Jin ${ }^{2}$ and Karim Oweiss ${ }^{1,3}$
}

\author{
Address: ${ }^{1}$ Electrical and Computer Engineering Dept., Michigan State University, East Lansing, MI 48824, USA, ${ }^{2}$ Computer Science and \\ Engineering Dept., Michigan State University, East Lansing, MI 48824, USA and ${ }^{3}$ Neuroscience Program, Michigan State University, East Lansing, \\ MI 48824, USA \\ Email: Seif Eldawlatly* - eldawlat@egr.msu.edu \\ * Corresponding author
}

from Seventeenth Annual Computational Neuroscience Meeting: CNS*2008

Portland, OR, USA. 19-24 July 2008

Published: II July 2008

BMC Neuroscience 2008, 9(SuppI I):PI9 doi:I0.I I86/I47I-2202-9-SI-PI9

This abstract is available from: http://www.biomedcentral.com/I47I-2202/9/SI/PI9

C) 2008 Eldawlatly et al; licensee BioMed Central Ltd.

\section{Introduction}

Recent technological advances in microfabrication of high-density multi-electrode arrays (MEA) permitted simultaneous recording of a few hundreds of neurons, and thus paved the way to investigate the dynamics of distributed neural circuits. Identifying functional connectivity between these simultaneously recorded neurons is important to understand how the brain orchestrates information processing when performing complex motor tasks.

There has been substantial amount of work geared towards inferring functional connectivity between cortical neurons. Partial correlation analysis and partial spectral coherence are among the known methods to achieve such objective. They are, however, based on the assumption that the recorded neurons are linearly correlated. In this paper, we demonstrate the utility of Dynamic Bayesian Network (DBN) in inferring this functional connectivity. Based on a conditional dependence on the neuron's parent history, DBN can be used to infer causal relationships between neurons without assuming linearity. DBN can also discriminate between direct and indirect connections that may exist between any pair-wise neurons.

\section{Methods}

Two models were used to simulate firing patterns of spiking neurons. The first was an inhomogeneous Poisson model where the firing probability of a given neuron depended on the background rate, an extrinsic covariate, and the neuron's own firing history and that of other neurons connected to it. By systematically varying the synaptic strength with other neurons, the model accounted for the discharge variability typically observed in real cortical neurons. The second model was a leaky integrate-and-fire (LIF) in which variations in neuronal biophysical properties such as the membrane potential and synaptic delays could be incorporated.

\section{Results}

The results demonstrate that DBN can correctly infer all the connections in networks of 16 neurons with both excitatory and inhibitory synapses. We also investigated the performance when two different types of inputs were present: a continuous external stimulus and a discrete input from an unobserved pre-synaptic neuron. In both cases, DBN correctly inferred bi-directional connections between pairs of neurons receiving the same input.

We also investigated the effect of varying biophysical properties on the accuracy in the LIF model. We observed that a mismatch between the chosen spike trains' bin width and the synaptic delay leads to degraded performance. Adjusting the sampling bin width based on known physiological characteristics of the brain area under study can overcome this problem.

\section{Acknowledgements}

This work was supported by NIH grant \# NS054 I 48-0IAI. 